\title{
Photoelectron Spectroscopy for Chemical Analysis
}

\author{
Håkan Rensmo and Hans Siegbahn*
}

\begin{abstract}
Photoelectron spectroscopy started its modern development in the fifties based on techniques for studies of nuclear decay. Since then, photoelectron spectroscopy has undergone a dramatic expansion of application and is now a prime research tool in basic and applied science. This progress has been largely due to the concomitant development of photon sources, sample handling and electron energy analyzers. The present article describes some of the salient features of modern photoelectron spectroscopy and its applications with particular emphasis on energy relevant issues.
\end{abstract}

Keywords: Chemical analysis · Photoelectron spectroscopy

\section{Introduction}

The development of photoelectron spectroscopy (PES) spans a time period of more than a century and it may be appropriate to give a few introductory historical notes. Following the discovery of the photoelectric effect by Heinrich Hertz in $1887^{[1]}$ and subsequent experimental work by others, ${ }^{[2,3]}$ Einstein laid the foundation in his famous paper of $1905^{[4]}$ for its interpretation in terms of the photon concept expressed thus:

$$
\mathrm{E}_{\text {kinetic }}=\mathrm{hv}-\mathrm{E}_{\text {binding }}
$$

where $E_{k i n t i c}$ is the kinetic energy of the ejected photoelectron, hv the photon energy and $E_{\text {binding }}$ the binding energy of the electron in the material.

During the decades after Einstein's paper, experiments on the photoelectric effect developed essentially along two independent lines. The first line was concerned with photoelectric yield phenomena near threshold. In the other line of research investigations were made on $\mathrm{X}$-ray excited photoelectron spectra. ${ }^{[5]}$ These latter investigations were however hampered by severe experimental difficulties concerning resolution and electron detection among other things. As a consequence of these experimental shortcomings, the physical and chemical applications of photoelectron spectroscopy where high resolution and well-defined line structures are required could not be realized.

${ }^{*}$ Correspondence: Prof. H. Siegbahn Department of Physics and Astronomy Uppsala University

Box 516

SE75120 Uppsala, Sweden

E-mail: hans.siegbahn@physics.uu.se
It was not until the fifties, when techniques developed for nuclear spectroscopy, in particular internal conversion in radioactive decay, that the energy resolution of electron spectrometers reached the performance for the ensuing development of photoelectron spectroscopy. ${ }^{[6,7]}$ It was then found that essentially symmetric photoelectron lines could be resolved from the high kinetic energy edges of the electron loss continua ( $c f$. Fig. 1). Moreover, the use of softer characteristic X-radiation, such as $\mathrm{AlK} \alpha(\mathrm{hv}=1486.6 \mathrm{eV})$ and $\mathrm{MgK} \alpha(\mathrm{h} v=$ $1253.6 \mathrm{eV}$ ) was found advantageous from the point of view of signal to background ratio as well as energy resolution. This development initiated comprehensive studies of electron energy levels of a large number of elements in the periodic system ${ }^{[8]}$ in many cases leading to substantial revisions of previously accepted values. It was also observed in the course of these measure-

ments that core electron lines from an element shift in energy upon change in chemical state of the atom. ${ }^{[9]}$ Another important characteristic of the spectral lines is that they originate from the outermost surface layers of condensed phase samples, corresponding to the photoelectron inelastic mean free path as displayed in Fig. 2. Thus, the various atomic components of a molecular species from a surface layer of molecular dimensions could be analyzed by means of its core electron spectrum and recorded small chemical shifts could be used for information on bonds and charge distributions. [10]

During the past decades, the field has literally exploded with on the order of ten thousand publications annually related to the use of photoelectron spectroscopy. Several important new advancements in the techniques have contributed to this development. As indicated in the above

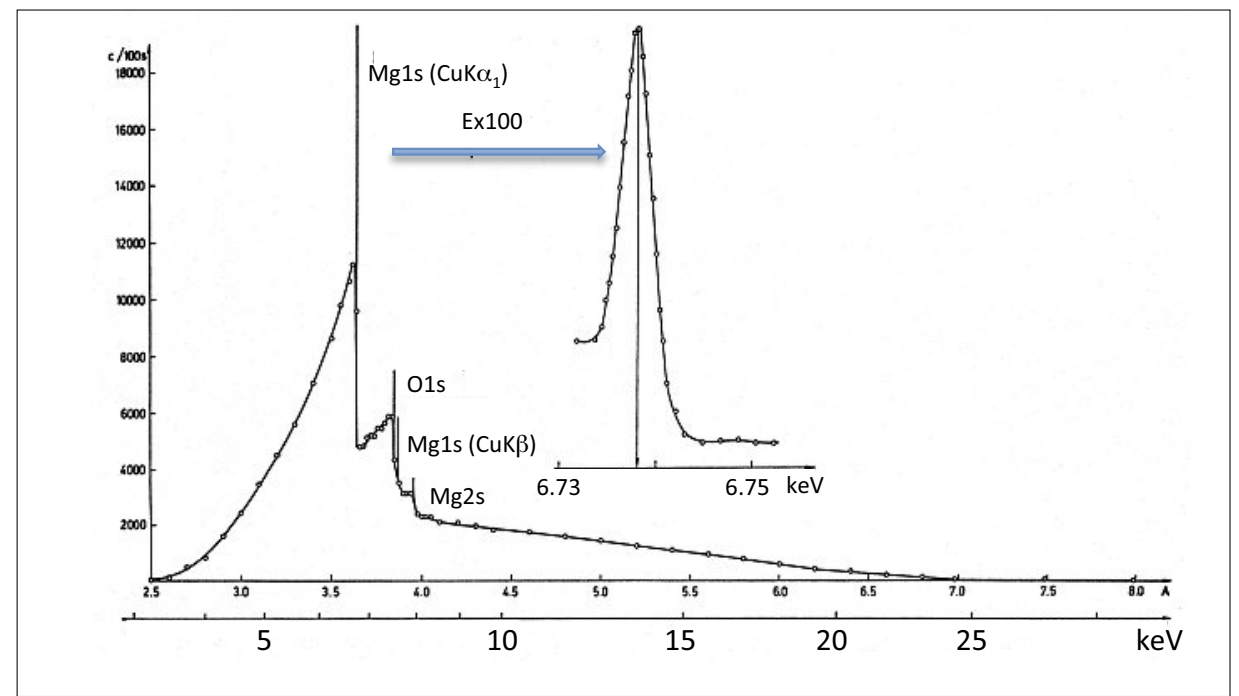

Fig. 1. Early photoelectron spectrum of MgO excited with a copper anode X-ray tube and measured with a magnetic field electron spectrometer, displaying electron energy loss continua on a bremsstrahlung background extending up to the tube acceleration voltage of $25 \mathrm{kV}$. The inset shows resolution of the leading edge elastic $\mathrm{Mg} 1 \mathrm{~s}\left(\mathrm{CuK} \alpha_{1}\right)$ photoelectron line. ${ }^{[8]}$ 


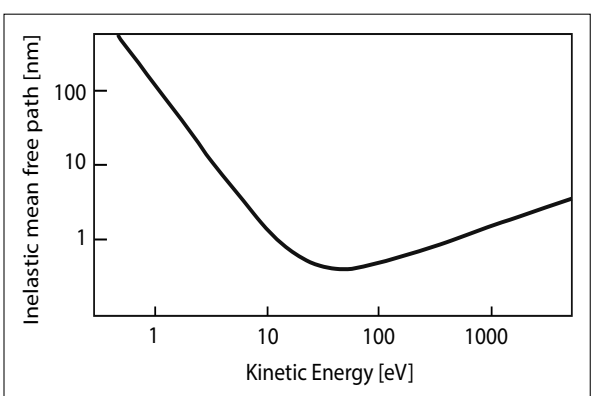

Fig. 2. Electron inelastic mean free path as a function of kinetic energy.

description of the early history, there are basically three ingredients for furthering of the techniques and broadening of the applications: the photon source, the sample handling and the electron energy analyzer. As for the first of these items, starting in the seventies, the development of largescale facilities based on synchrotron radiation has had a dramatic impact on the field. The use of synchrotron radiation for photoelectron spectroscopy implies a number of important advantages compared to characteristic lab sources, such as AlK $\alpha$. Not only can the photon be tuned to desired values within wide ranges, to study e.g. resonance phenomena, intensities can also be made orders of magnitude larger via insertion devices in the storage rings. An important aspect is also that the emitted X-radiation is polarized in the plane of the storage ring. This, naturally, has widened the scope substantially in performing different experiments using photoelectron spectroscopy. This has not meant, however, that the use of conventional X-ray sources has become obsolete; AlK $\alpha$-based photoelectron spectrometers are still abundant home-lab instruments in materials science institutions.

Concerning electron spectrometers and detectors, the early magnetic instruments were soon replaced by devices based on electrostatic fields, such as hemispherical analyzers, where the electron kinetic energies are dispersed along a focal plane al-

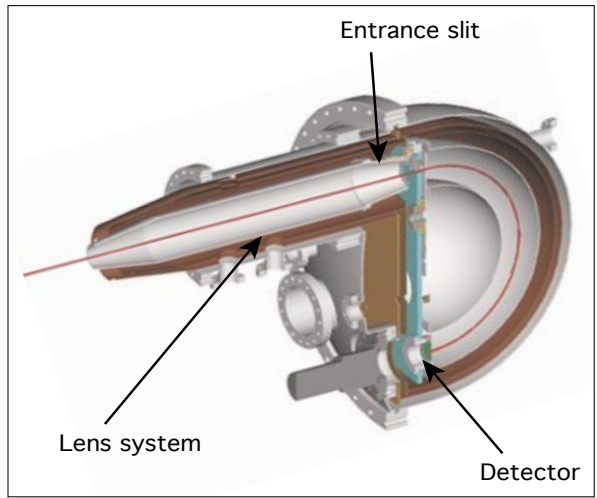

Fig. 3. Electron trajectory in an electrostatic field hemispherical electron energy analyzer. (Courtesy of VG Scienta) lowing for multichannel detection. These instruments are equipped with input lens systems for efficient transport of the photo-ejected electrons into the analyzer (cf. Fig. 3). Other types of analyzers are based on time-of-flight tubes allowing for detection over large solid angles as well as angle-resolved measurements. ${ }^{[11]}$ Development of analyzer technology has, in parallel with that of the photon sources, resulted in a very powerful boost in terms of the science that can efficiently be performed with photoelectron spectroscopy.

Being a technique based on electron analysis puts particular demands on the sample preparation. The energy analysis in the electron analyzer requires at least high vacuum on the order of $10^{-5}$ mbar or lower. This implies that samples will have to be introduced into and maintained in the analysis chamber using special vacuum lock devices. Prior to the introduction of the sample, it is normally subject to preprocessing e.g. in special preparation chambers or under inert gas in a glovebox using transfer systems. Depending on the nature of the sample and experiments to be performed the vacuum conditions in the analysis chamber may vary by many orders of magnitude. For example, in order to fully exploit the inherent surface sensitivity for carefully controlled studies of the outermost atomic layers of single crystal surfaces, it is necessary to work in the ultra high vacuum regime (below $10^{-10} \mathrm{mbar}$ ) in the sample region.

For gaseous or liquid phase samples, on the other hand, the latter having vapor pressures set by temperature, sample pressures may be in the mbar region. For such systems it is necessary to introduce extra pumping stages for pressure reduction close to the sample and further into the analyzer region to avoid severe losses of photoelectrons due to inelastic scattering. The techniques for sample handling have been continuously developing to expand the limits of systems amenable for study. For instance, it is now possible, using a combination of pumping and electron lens systems (so-called Near Ambient Pressure Photoelectron Spectroscopy), to study catalytic processes at solid surfaces under realistic conditions as well as liquid water systems at room temperature conditions (up to 30 mbar). ${ }^{[12-15]}$

Another recent line of development to further widen the scope of photoelectron spectroscopy is to exploit higher photon energies for excitation, of the order of 5-10 keV, so called HAXPES (Hard X-ray Photoelectron Spectroscopy). ${ }^{[16]}$ As evidenced by Fig. 2, this implies a substantial increase in inelastic mean free path, which means that deeper layers of the sample can be probed. In turn, this opens possibilities to study multi-layer systems pertinent to real devices, such as solar cell structures. Moreover, it allows for variation in relative photoelectric cross sections of the constituent elements such that the electronic structure of selected atoms may be highlighted.

The experimental development of photoelectron spectroscopy has had an impact on many areas of science where the interplay between basic and applied research has been extremely successful. Studies of energy relevant materials is one such area. The aim of the present article is to give a few examples of current work using photoelectron spectroscopy, in particular focused on systems of relevance for energy efficient conversion and storage, $c f$. Fig. 4. The intention is furthermore to illustrate how the recent technical developments of the experimental techniques have provided new routes for the understanding of the structure/function relationship for these systems at an atomic level.

\section{Light to Electrical Energy Conversion - Heterojunction Solar Cells}

Nanostructure architectures have facilitated the evolution of strategies to design new-generation solar cells. ${ }^{[17,18]}$ Three major types of cells that have dominated research in recent years include (i) dyesensitized solar cells (DSSC), (ii) organic photovoltaic cells or bulk heterojunction (BHJ) photovoltaic cells, and (iii) quantum dot solar cells (QDSC). Solid state DSSC analogues with organic hole conductors have also been successful and partly merge the two former types. ${ }^{[19]}$ Moreover, the basic research on different nanostructures sensitized with quantum dot materials has led to an unforeseen development of organometal halide perovskite solar cells (e.g. $\mathrm{CH}_{3} \mathrm{NH}_{3} \mathrm{PbI}_{3}$ ). ${ }^{[20-22]}$ Power conversion efficiency attained with the hybrid organic-inorganic perovskite $\mathrm{CH}_{3} \mathrm{NH}_{3} \mathrm{PbX}$ $(\mathrm{X}=\mathrm{Cl}, \mathrm{I})$ has quickly exceeded $15 \%$, and currently both 3D-nanostructured designs as well as $2 \mathrm{D}$ thin film designs are investigated.

The conversion mechanism in different heterojunction solar cells starts with light absorption and is followed by charge injection and subsequent charge separation in the electron-conducting and hole-conducting materials. In all systems the mechanisms include diffusion and interfacial charge transfer reactions and the overall efficiency largely depends on the competition between different reactions in different time regimes. For traditional silicon and thin film solar cells the mechanism is generally described in terms of band bending, while for molecular dye or semiconductor sensitized nanoporous solar 


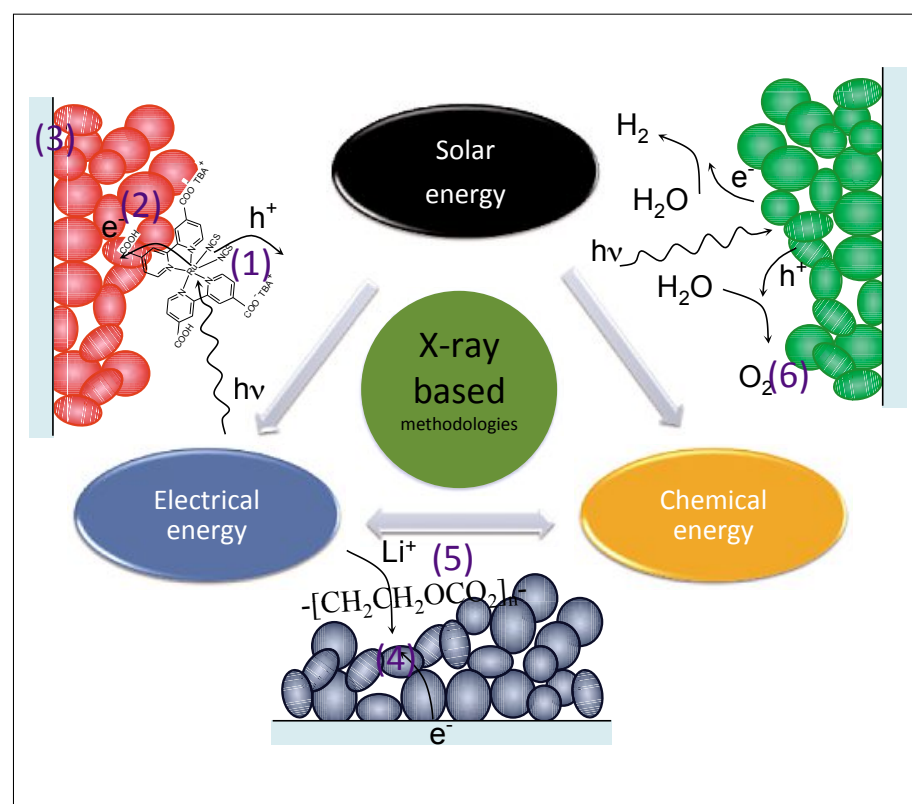

cells it involves the competition between the back-reaction of the electron from the semiconductor conduction band to the light absorber (or the hole-conductor) versus the successful reduction of the oxidized dye and subsequent hole-transport via the molecular material (electrolyte or hole-conductor). The overall function of a single heterojunction depends on basic electronic properties of the different materials such as molecular orbital compositions, band positions, light absorption properties and redox potentials, as well as on simple geometrical properties, such as position of specific linking groups and material mixing, important for connecting the materials. In real systems, however, when assembling the materials the relative material matching, both in terms of molecular orientations or material interfacial composition and electronic structures adjust due to the interactions between them and the combined molecular function may therefore be altered with respect to the separate materials. Photoelectron spectroscopy is a prime tool for obtaining information on the interfacial characteristics and below we give some examples from such investigations and also describe some future directions.

\subsection{Structure of Solar Cell Interfaces - From Model Surfaces to Real interfaces}

As mentioned above, charge transfer reactions at interfaces are often largely dependent on the detailed structure of the interface. In DSSC the sensitizers are usually adsorbed to the semiconductor surface through an anchoring group. The function of this group is to give high stability, favorable molecular ordering and to facilitate charge transfer. Carboxylic groups functionalizing different organic and metalorganic chromophores have shown such
Fig. 4. Schematic picture exemplifying some sustainable energy systems including dye-sensitized solar cells, lithium ion batteries and catalysis/photocatalysis. Different materials and interfaces are discussed in the text including (1) Cul/dye/ $\mathrm{TiO}_{2}$ interfaces, (2) dynamics at dye/TiO interfaces, (3) buried interfaces in conducting glasses, (4) lithium ion insertion in nanostructured $\mathrm{TiO}_{2}$, (5) SEl layers on lithiated graphite, (6) heterogeneous catalysis at platinum surfaces.

characteristics when adsorbed onto oxide substrate. PES has shown to be an efficient tool to investigate the binding configuration. For the understanding of complex molecular interfaces a common route has been to investigate different levels of complexity of model molecules. UHV investigations of formic acid adsorbed onto $\mathrm{TiO}_{2}$ (110) rutile reveal a chelating carboxylate binding structure ${ }^{[23]}$ and carboxylic functional groups attached to the 2,2'-bipyridine unit show a very similar carboxylate structure.[24] This conclusion was partly based on the fact that the O1s signal from the oxygen atoms in the carboxylic unit appears as a single peak shifted $1.6 \mathrm{eV}$ from the substrate oxygen peak. Further analysis has shown that this anchoring mechanism prevails for full dye adsorption on $\mathrm{TiO}_{2}$ surfaces as sketched in Fig. 5 for the large metal-organic chromophore $\mathrm{RuL}_{2}(\mathrm{NCS})_{2}$, where $\mathrm{L}$ is biisonicotinic acid containing carboxylic anchor groups. With these results in mind the O1s photoelectron spectra for even more complex structures can be analyzed. Fig. 6 shows the O1s and

S2p spectra of $\mathrm{RuL}_{2}(\mathrm{NCS})_{2}$ attached to a nanostructured $\mathrm{TiO}_{2}$ anatase surface. The figure shows how the O1s and S2p levels develop upon evaporation of the $\mathrm{p}$-doped material CuI. ${ }^{[25]}$ The O1s spectrum of the dye on $\mathrm{TiO}_{2}$ (second from the bottom in Fig. 6, left) contains three peaks identified as the $\mathrm{TiO}_{2}$ substrate oxygen (1), doublebonded and surface-adsorbed oxygen (2) as well as $\mathrm{OH}$ groups (3). Based on the discussion above it can be inferred that the RuL (NCS) dye may coordinate to an anatase $\mathrm{TiO}_{2}$ surface through two of the four carboxylic groups ( $c f$. Fig. 5), which would ideally yield an intensity ratio of three between peak 3 and 2. In our case, we find that the ratio is about 1.9 directly after sensitization. The deviation from the expected value indicates a mixture of adsorption structures at the nanostructured anatase $\mathrm{TiO}_{2}$ surface. The mixed adsorption configuration is further supported by the S2p spectrum (bottom Fig. 6, right) in which two spin-orbit split doublets $(1,2)$ are required to fit the measurements of the dye molecule at the oxide surface.

Proceeding from a dye-sensitized surface to a buried interface representing a functional configuration is an experimental challenge. To meet this challenge two different routes may be taken: (i) following the interfacial chemistry step-wise during formation and (ii) forming the complete interfacial structure and then using hard X-ray photoelectron spectroscopy (HAXPES) to investigate the buried interface. The former route entails preparation and analysis of the materials in an ultra high-vacuum environment while the latter requires high photon energies, and thus detection of high kinetic energy photoelectrons, to achieve an increased bulksensitivity for condensed samples.

An example of the first of these approaches is given by the spectral evolutions for the $\mathrm{TiO}_{2} /$ dye $\left(\mathrm{RuL}_{2}(\mathrm{NCS})_{2} /\right.$ hole conductor(CuI) interface as shown in Fig. 6. The CuI was deposited stepwise in situ

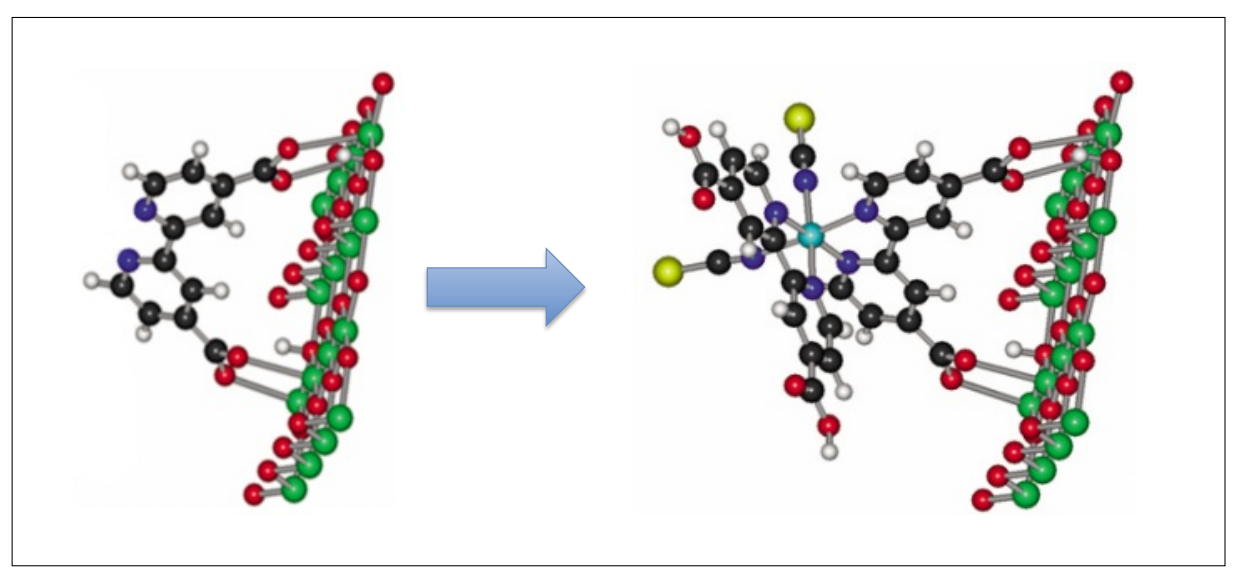

Fig. 5. Geometries for RuL ${ }_{2}(\mathrm{NCS})_{2}$ adsorption on a $\mathrm{TiO}_{2}$ surface. Left: adsorption geometry of the dye ligand (biisonicotinic acid) based on the $\mathrm{O} 1 \mathrm{~s}$ photoelectron spectrum showing only a single peak for the ligand. Right: adsorption of the full dye molecule. 


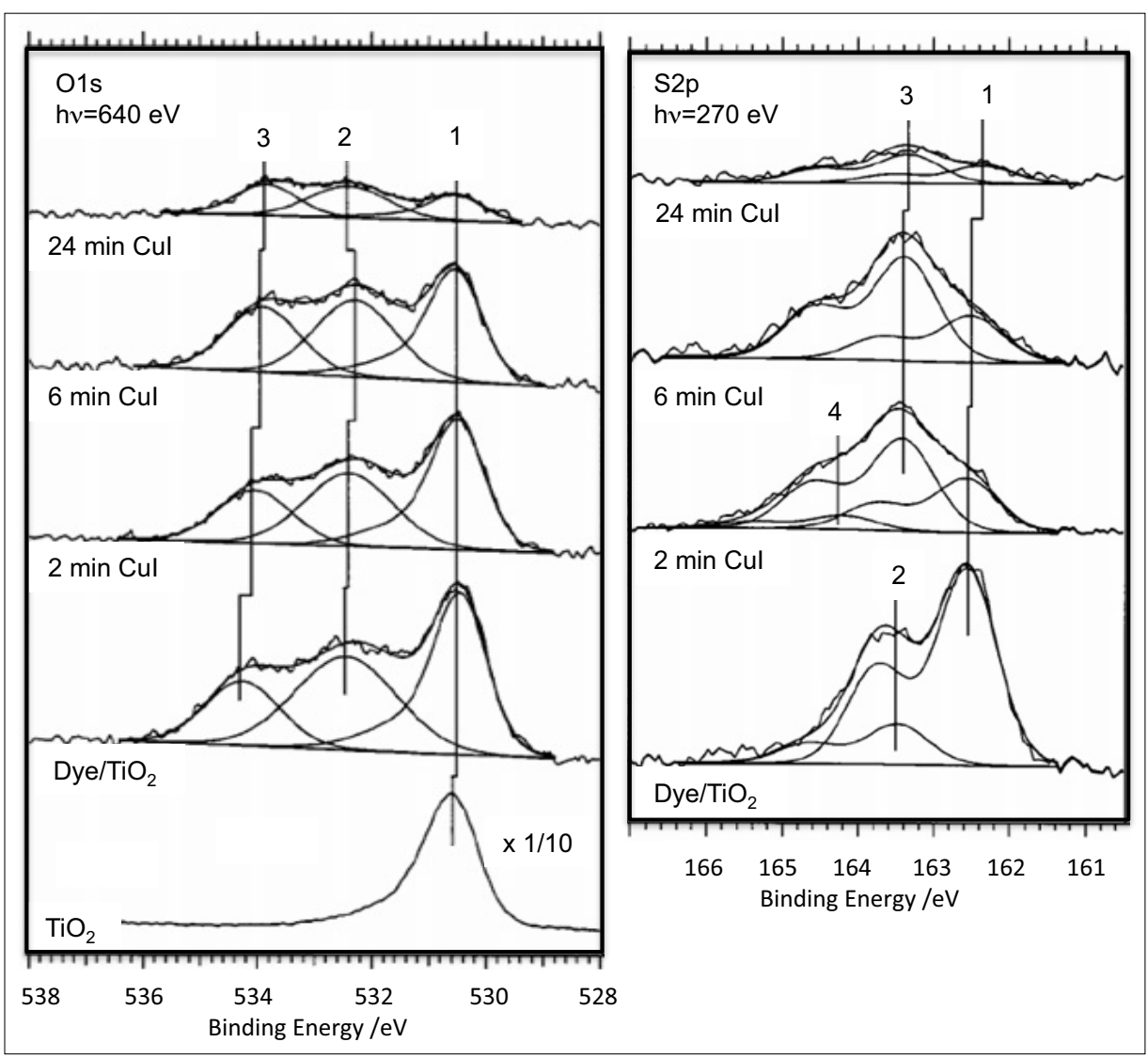

Fig. 6. O1s (left) and S2p (right) photoelectron spectra for a multilayer solar cell structure consisting of semiconductor $\left(\mathrm{TiO}_{2}\right)$ /dye $\left(\mathrm{RuL}_{2}(\mathrm{NCS})_{2} /\right.$ hole conductor (Cul) at different deposition times of Cul. The photon energies were chosen to give similar probing depth for O1s and S2p. Peaks 2 and 3 in the O1s spectra are due to the dye anchoring groups, the relative intensities of which change with increasing Cul layer thickness indicating effects on the dye/semiconductor bonding. Peaks 3 and 4 in the S2p spectra are due to Cu-NCS interactions. ${ }^{[25]}$

onto the $\mathrm{RuL}_{2}(\mathrm{NCS})_{2} / \mathrm{TiO}_{2}$ interface and the dye- $\mathrm{TiO}_{2}$ and dye-CuI interaction was monitored as function of the amount of CuI. A clear evolution of the peaks is observed. Specifically, a direct interaction between the dye NCS groups and the $\mathrm{CuI}$ is observed in terms of the appearance of a new feature (3) in the S2p spectra (Fig. 6, right) indicating the formation of $\mathrm{Cu}-\mathrm{SCN}$ bonding. At the same time, effects on the carboxylate anchoring can be observed in the O1s spectra (Fig. 6, left) indicated by changes between the ratios of peak 3 and 2 .

An example of the latter of the two approaches above to investigate a buried interface, i.e. the use of high photon energy with HAXPES, is demonstrated by the investigation of metal oxide thin films. $\mathrm{ZnO} / \mathrm{Ti}$ based thin films find use in several areas. For example, Ti forms an ohmic contact to $\mathrm{ZnO}$. In Fig. 7 we demonstrate the measurement and annealing of a Mo/ $\mathrm{ZnO} / \mathrm{Ti} / \mathrm{ZnO}$ sample with nominal thicknesses of $7 \mathrm{~nm}(\mathrm{Mo}), 5 \mathrm{~nm}(\mathrm{ZnO}), 4 \mathrm{~nm}$ (Ti) and $50 \mathrm{~nm}(\mathrm{ZnO})$ formed by sputter deposition. Employing the increased probe-depth at $4000 \mathrm{eV}$ photon energy one can quantify the extent of $\mathrm{ZnO}$ reduction in the as-deposited $\mathrm{ZnO} / \mathrm{Ti} / \mathrm{ZnO}$ stack and follow how the composition of the buried interface changes upon annealing as well as get an insight into the band-bending at such an interface. ${ }^{[26]}$

\subsection{Resonant Photoelectron Spectroscopy for Studies of Ultrafast Electron Transfer}

The use of synchrotron radiation implies that photoelectron spectra from a given sample can be studied over tunable ranges of the exciting photon energy. In particular, the photon energy may be tuned in resonance with X-ray absorption transition energies, resonant photoelectron spectroscopy (RPES). This mode of operation can be used in order to study ultrafast phenomena, such as electron transfer across an interface. For the dye-sensitized solar cell (DSSC), such experiments have been performed in order to estimate the transfer time from the dye to the semiconductor. A model system, consisting of the dye $\left(\mathrm{RuL}_{2}(\mathrm{NCS})\right.$, ligand $(\mathrm{L}=$ biisonicotinic acid) adsorbed on nanostructured anatase $\mathrm{TiO}_{2}$ was used to simulate the real solar cell dye/semiconductor interface. The injection process studied was:

$\mathrm{N} 1 \mathrm{~s}(\mathrm{~L}) \rightarrow \mathrm{LUMO}(\mathrm{L}) \rightarrow \mathrm{TiO}_{2}$

Although this process is not identical to that of the real solar cell, the important injection step is the second in the above scheme, which may be assumed very similar in character. In estimating the injection time, the lifetime of the N1s core hole can then be used to 'clock' the process. ${ }^{[27,28]}$ This lifetime has been measured previously to be $6 \mathrm{fs}$. The argument then runs as follows, $c f$. Fig. 8 and 9. The N1s X-ray absorption spectrum of the adsorbed ligand contains three clearly resolvable peaks corresponding to transitions from N1s to unoccupied states of the ligand. The lowest of these (LUMO) lies below the conduction band edge of the semiconductor and can thus not inject the excited electron into the semiconductor. The next two, LUMO+1 and $\mathrm{LUMO}+2$, lie above the edge and are candidates for electron injection.

The appearance of the photoelectron spectra recorded over the photon energy range of the X-ray absorption spectrum will depend on whether the excited electrons have been injected or not within the

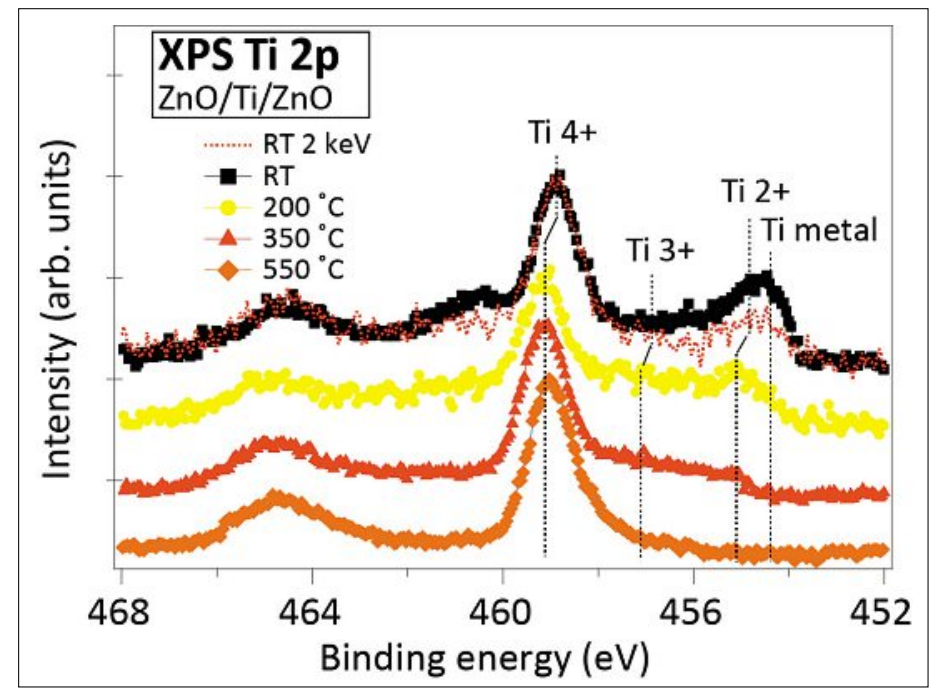

Fig. 7. A Ti2p

HAXPES study of a buried Ti interface in a $\mathrm{ZnO} / \mathrm{Ti} / \mathrm{ZnO}$ sample upon annealing. The vertical dotted lines mark the binding energy of Ti oxidation states $(4+, 3+, 2+$ $0)$. The initially weak metallic component is almost completely lost after annealing at $200^{\circ} \mathrm{C}$. Based on such data a complete mapping of the depth profile and interface chemistry could be accomplished. ${ }^{[26]}$ 


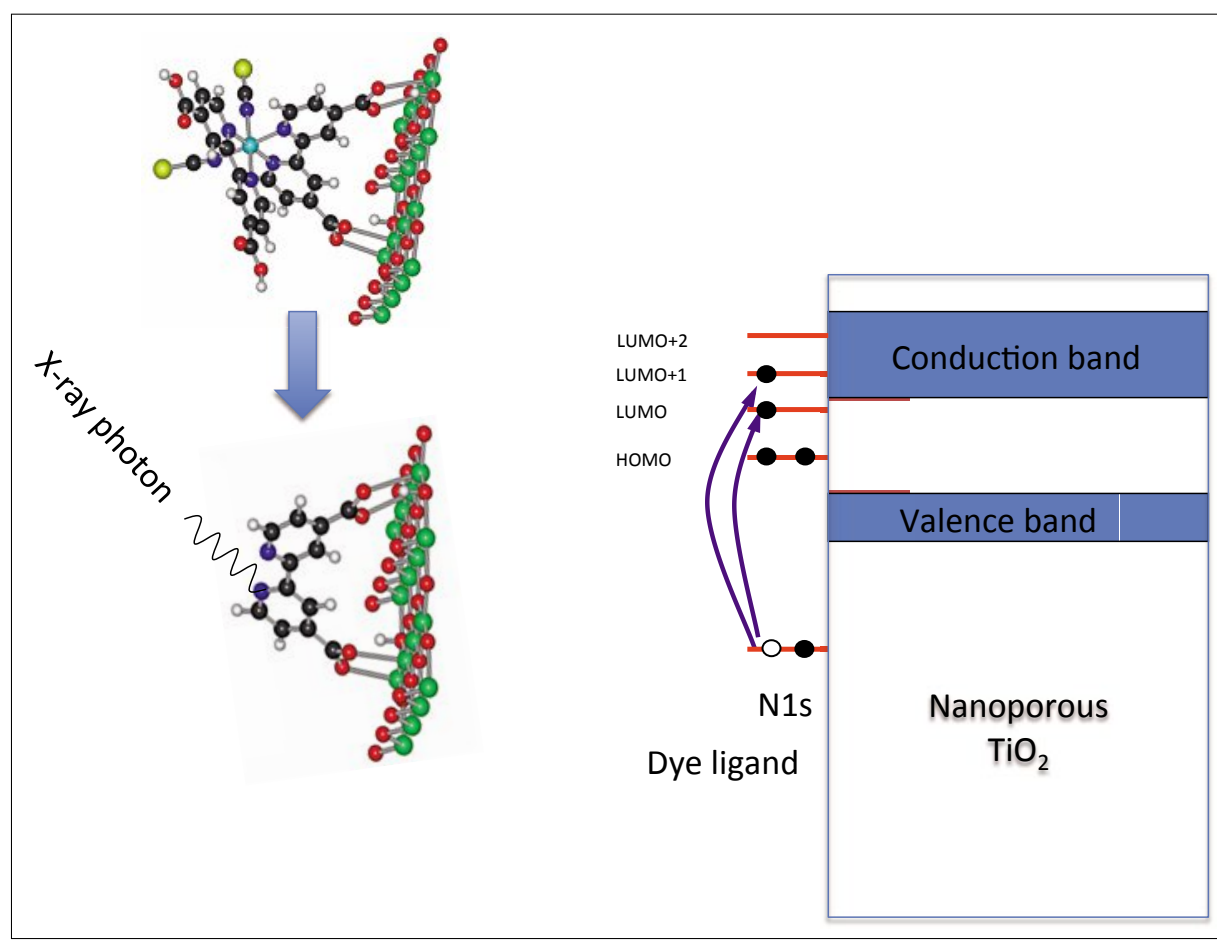

Fig. 8. Model study of electron injection from a dye into nanoporous $\mathrm{TiO}_{2}$. The dye molecule is simulated by its anchoring ligand, biisonicotinic acid, and photoinjection is performed by excitation from the ligand $\mathrm{N} 1 \mathrm{~s}$ level into $\mathrm{LUMO}+1$ and +2 states above the $\mathrm{TiO}_{2}$ conduction band edge.

time frame of the N1s core hole. Fig. 9 shows a comparison between the X-ray absorption spectrum and the intensity of the HOMO photoelectron peak (N1s RPES) of the ligand molecule as a function of photon energy. As can be seen, the LUMO+1 and LUMO+2 resonances are absent in the resonant photoelectron results which means that the electrons excited to these states have indeed been injected into the semiconductor on a time scale significantly shorter than the N1s core hole lifetime, i.e. ultrafast fs or below.

\section{Electrical Energy to Chemical Energy Conversion - Li Batteries}

An attractive route to reversibly store energy is electrochemical insertion or intercalation of ions into host materials. ${ }^{[29]}$ Some material combinations now perform excellently for small portable electronics but there is still much room to improve the kinetics and energy density for high power applications. To solve these problems alloys with high Li density and nanostructured materials are currently of applied and fundamental interest. Examples include Si and nanostructured oxides. ${ }^{[30-32]}$ Lithiumbased batteries based on these ideas allow for very high energy density in terms of weight and volume being based on lithium which is the smallest and lightest metal. Many commercial batteries use a carbon anode and a lithium-cobalt-nickel oxide cathode that can store about $130 \mathrm{mAh} / \mathrm{g}$. In general, however, the lithium-based systems consist of a set of chemistries that can be tuned to optimize a specific application but where many of the fundamental properties are similar. It is today generally realized, however, that not only the bulk material structure but also the host material surface and the solid/electrolyte interphase (SEI) need to be better understood on an atomic level to improve the performance of lithium ion batteries in terms of selfdischarge, safety and cycling stability.

Photoelectron spectroscopy is well suited to investigate surfaces and layers with $\mathrm{nm}$ dimensions such as those described for the lithium ion batteries above. This refers to determination of stoichiometries and depth profiles as well as to the understanding of the character of electronic states in both crystalline and non-crystalline materials. However, the investigation of liquid electrolyte interfaces of interest for $\mathrm{Li}$-ion batteries has required methodology development involving preparation, sample environment and vacuum design to match with the constraints set by traditional photoelectron spectroscopy.

One experimental challenge of using photoelectron spectroscopy for the investigation of interfaces that have been electrochemically modified for charge storage of lithium in the metallic oxidation state is their sensitivity to oxygen and water. This is a sensitivity that for most anode materials is easily understood from the electrochemical potential used for its preparation. To solve such problems different methodologies could be used, e.g. ex situ sample preparation combined with transfer systems allowing for controlled

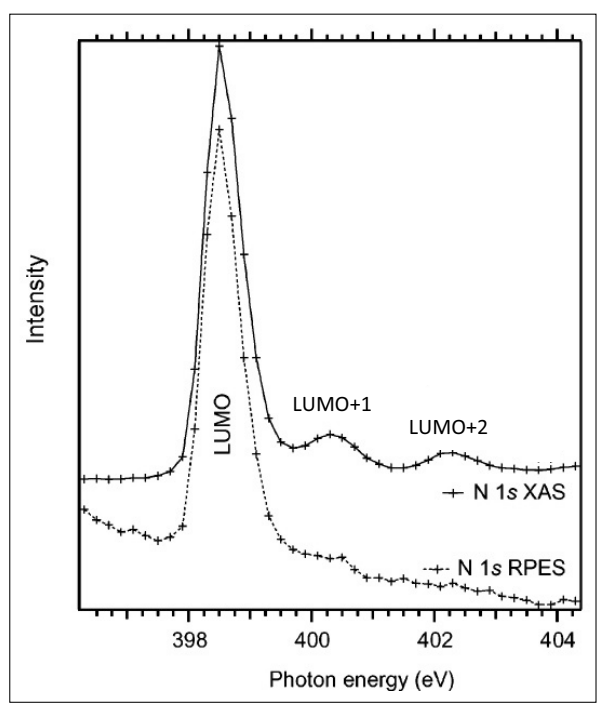

Fig. 9. Comparison between the N1s X-ray absorption spectrum (N1s XAS) of the biisonicotinic anchoring group and the intensity of the highest occupied molecular orbital (HOMO) (N1s RPES). ${ }^{[27]}$

transfer to the photoelectron spectroscopy analysis chamber or an in situ vacuum/ sample configuration design that allows for high sample pressure during the photoelectron spectroscopy measurements. One early example of the first method is shown in Fig. 10. ${ }^{[33]}$ The electrochemistry is here performed in the preparation chamber in an atmosphere set by the vapor pressure of the electrolyte and controlled by a careful pumping procedure. Subsequently, the sample can be introduced for analysis, while the electrolyte bath is retracted into a separately sealed compartment in the preparation chamber. The process can be repeated at different electrode potentials and the resulting surface electrochemistry thus monitored. Investigation of the SEI using photoelectron spectroscopy has since then been numerous and many of these investigations have utilized different kinds of systems to transfer the samples from a glove-box to the analysis chamber without water/oxygen exposure. ${ }^{[34]}$

The electrochemical insertion of $\mathrm{Li}$ ions into a $\mathrm{TiO}_{2}$ electrode may be studied using PES as shown in Fig. 11. ${ }^{[35]}$ The Ti2p spectrum was recorded for different values of $\mathrm{x}$, where $\mathrm{x}$ is defined by the reaction:

$\mathrm{xLi}^{+}+\mathrm{TiO}_{2}+\mathrm{xe}^{-} \rightarrow \mathrm{Li}_{\mathrm{x}} \mathrm{TiO}_{2}$

The electrodes were here prepared ex situ under inert gas in a glovebox and subsequently inserted into the photoelectron spectrometer via a transfer system. As seen from Fig. 11 (left), with increasing x-values a prominent peak at low binding energy evolves, whose intensity is proportional to $\mathrm{x}$. The position of this peak is consistent 


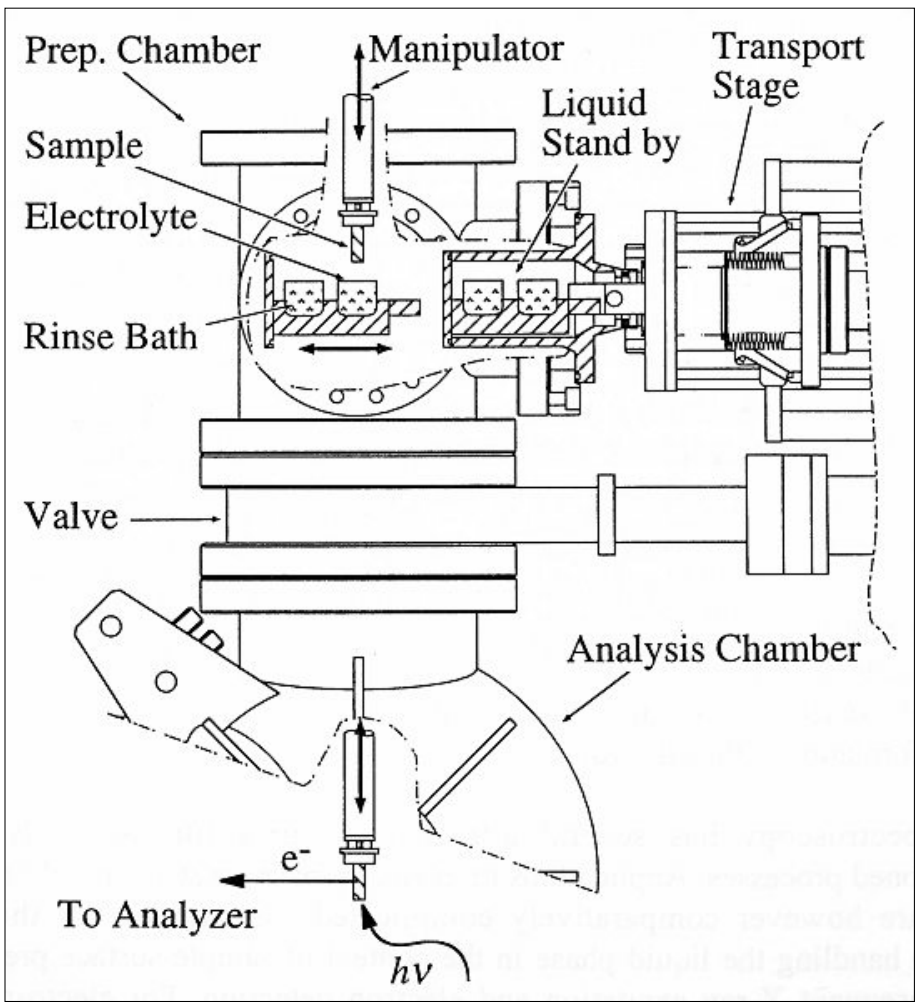

Fig. 10. Experimental arrangement for photoelectron spectroscopy of electrochemical interfaces. The electrochemistry is performed in the upper chamber by means of electrolyte baths that can be introduced on a movable holder. After the electrochemical preparation the sample electrode may be introduced into the analysis chamber while the electrolyte baths are retracted into a separate standby chamber. The full electrochemical cycling may be performed by repeating the transfer between the preparation and analysis chambers. ${ }^{[3]}$ shown in Fig. 12 where, on the left is the bandgap region at $\mathrm{x}=0$, i.e. with no $\mathrm{Ti}^{3+}$ sites present and, on the right recorded at $\mathrm{x}=0.5$. The latter spectrum clearly shows the resonant behavior expected from the discussion above, confirming the Ti3d character of the localized states.

Recording of the O1s spectra corresponding to the different $\mathrm{x}$-values of Fig. 11 , shows the growth of a peak at the high binding energy side of the peak associated with the $\mathrm{TiO}_{2}$ electrode material ( $c f$. Fig. 11 (middle)). Such a high binding energy feature is not directly a consequence of the insertion process ${ }^{[33,35]}$ but is one of the first photoelectron-spectroscopic results showing the formation of an SEI layer from the reduction of the electrolyte. The SEI on battery materials contains reduced electrolyte components and often has a thickness in the nm range. A recent example is demonstrated in Fig. 13 where the carbon signal for lithiated graphite has been measured using a range of photon energies. ${ }^{[36]}$ As can be seen, the low binding energy peak originating from the graphite-based substrate is with an oxidation number + III for the $\mathrm{Ti}$ atom. The growth of this Ti2p feature is accompanied by the appearance of a peak in the band-gap above the valence band edge, cf. Fig. 11 (right). The experimental data is interpreted in terms of localized states centered on the Ti atoms filled by the electrons associated with the insertion of the Li ions. The nature of these localized states as of Ti3d character may be inferred by means of resonant PES, i.e. by means of measurement of the bandgap peak while scanning over the Ti2p X-ray absorption edge $(2 \mathrm{p}$ $\rightarrow 3 \mathrm{~d}$ ). Thus the direct PES process of the bandgap peak is:

$$
2 \mathrm{p}^{6} 3 \mathrm{~d}^{1} \rightarrow 2 \mathrm{p}^{6} 3 \mathrm{~d}^{0}
$$

While the resonant PES process leading to the same final state can be described:

$$
2 p^{6} 3 d^{1} \rightarrow 2 p^{5} 3 d^{2} \rightarrow 2 p^{6} 3 d^{0}
$$

There are thus two contributions to the Ti3d bandgap peak in this resonant measurement. The contribution from the first, direct, process may be regarded as closely constant over the energy interval of the $\mathrm{X}$-ray absorption spectrum. The contribution from the second process, however, is dependent on the number of electrons excited into unoccupied $3 \mathrm{~d}$ states, hence a direct measure of the X-ray absorption spectrum for the $\mathrm{Ti}^{3+}$ atomic sites. The result of the resonant PES measurements is

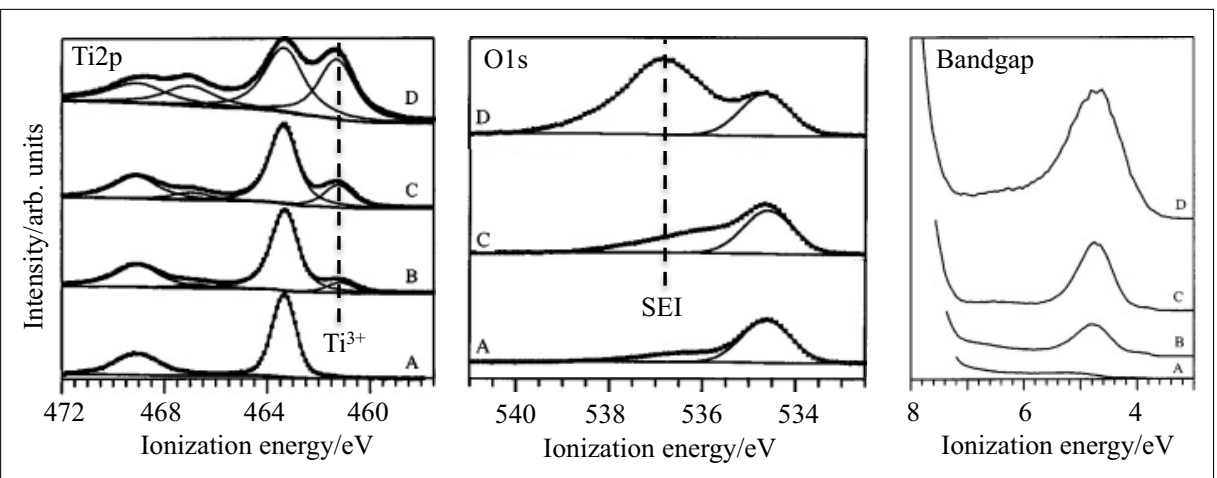

Fig. 11. Ti2p (left), O1s (middle) and bandgap photoelectron spectra as a function of different $\mathrm{X}$-values of $\mathrm{Li}$ intercalation into nanostructured $\mathrm{TiO}_{2}(\mathrm{~A}: \mathrm{x}=0 ; \mathrm{B}: \mathrm{x}=0.11 ; \mathrm{C}: \mathrm{x}=0.24$; $\mathrm{D}: \mathrm{x}=0.5)^{[35]}$

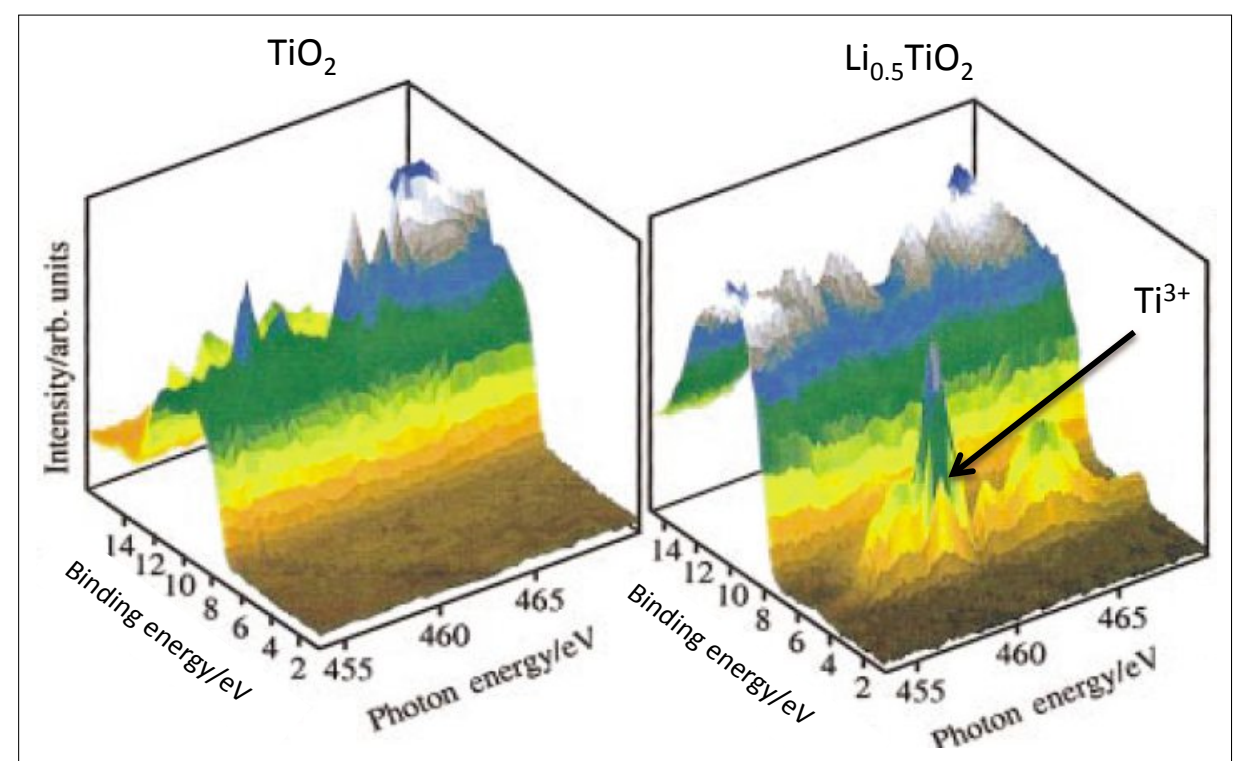

Fig. 12. Valence and bandgap photoelectron spectra of nanostructured $\mathrm{TiO}_{2}$ (left) and $\mathrm{Li}_{0.5} \mathrm{TiO}_{2}$ (right) recorded across the photon energy range of the Ti2p $\rightarrow$ Ti3d absorption resonance. ${ }^{[35]}$ 


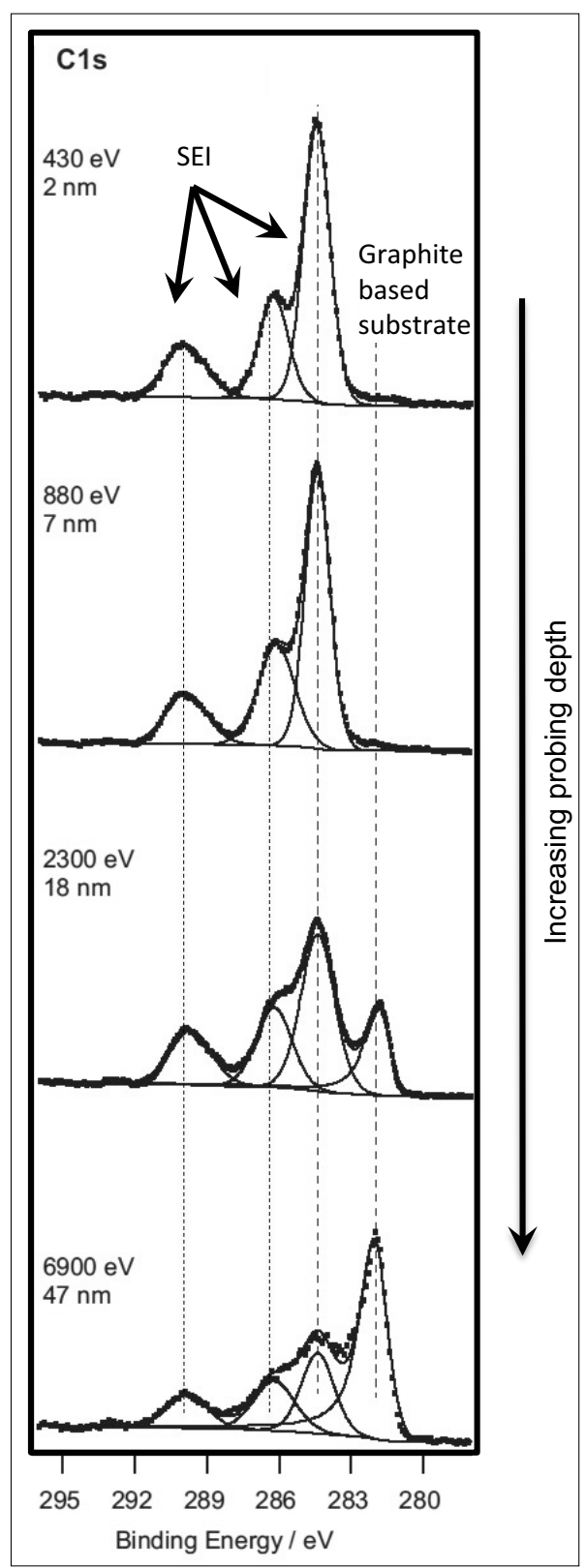

Fig. 13. Lithiated anode PES spectra using photon energies from the soft to hard X-ray (HAXPES) regimes. The excitation energies, along with the probing depth, are specified in the top left corner of the respective spectrum. The intensity of the graphite based substrate signal is increasing with excitation energy. Each spectrum was normalized by the area of the respective elemental signal. Increasing probing depth is indicated with an arrow. ${ }^{[36]}$

very weak in the spectrum measured with a low photon energy and thus with high surface sensitivity. The multitude of C1s peaks originate from the different compounds constituting the SEI interface. The spectrum measured with high photon energy, on the other hand, shows a dominating signal originating from the graphite-based substrate. This example demonstrates how the surface sensitivity can be varied in order to obtain a depth profile of the outermost surface layers yielding further insight into the chemistry of formation of the SEI.

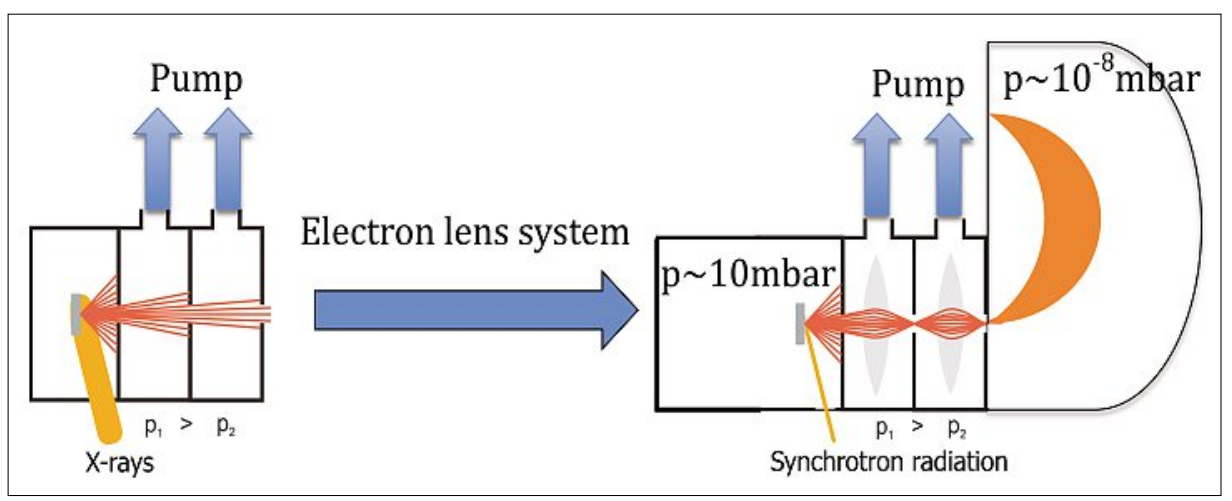

Fig. 14. Comparison of experimental setups for studies of ambient pressure substances with photoelectron spectroscopy. The setup on the left shows an early stage design with differential pumping to reduce the sample pressure along the electron trajectories. The setup on the right shows the combined use of differential pumping stages and electron lens systems allowing for substantially increased sample pressures while retaining photoelectron intensities. The use of this scheme is further advantaged using synchrotron radiation of high brilliance.

\section{Future Prospects of Photoelectron Spectroscopy; Near Ambient Pressure Techniques}

As described above, photoelectron spectroscopy is undergoing continuous development broadening the scope of applications in different scientific areas. In particular, substantial progress has been made in creating more realistic environments for samples being analyzed under vacuum conditions. This is crucial for the study of high vapor pressure substances, such as liquids or biological surfaces and processes dependent on the presence of such substances such as those at catalytic surfaces or electrodes. Already in the early phases of photoelectron spectroscopy such techniques were developed for studies of gases and liquids based on specially designed pumping arrangements of the sample region.[37] More recently, these techniques have advanced considerably due to combined utilization of synchrotron radiation and electron optics. ${ }^{[12-15]}$

The principle of this new technique is sketched in Fig. 14. In first generation setups (left) to reduce a sample vapor pressure load, one or a series of slits was introduced prior to the analyzer entrance associated with heavy intermediate, differential pumping. However, this also meant a loss rate of the photoelectrons of the same order of magnitude. If instead a lens system is introduced into the differential pumping stages (right), this loss rate can be largely overcome. Moreover, with high brilliance synchrotron radiation excitation, aperture sizes can be substantially reduced, which further reduces the gas load along the electron paths. The overall result of this design principle is that pressure gradients of eight to nine orders of magnitude can be maintained between the sample region and the spectrometer while still sufficient intensities of photoelectrons will be transmitted. The crucial region of intensity losses though is that between the sample surface and the first aperture, which has to be kept as small as possible while allowing access for the photon beam (in practice a distance on the order of one $\mathrm{mm}$ ).

Fig. 15 shows an example of the use of the setup in Fig. 14 for studies of catalytic processes, in this case oxidation of $\mathrm{CO}$ in an $\mathrm{O}_{2}$ atmosphere on a heated single crystal

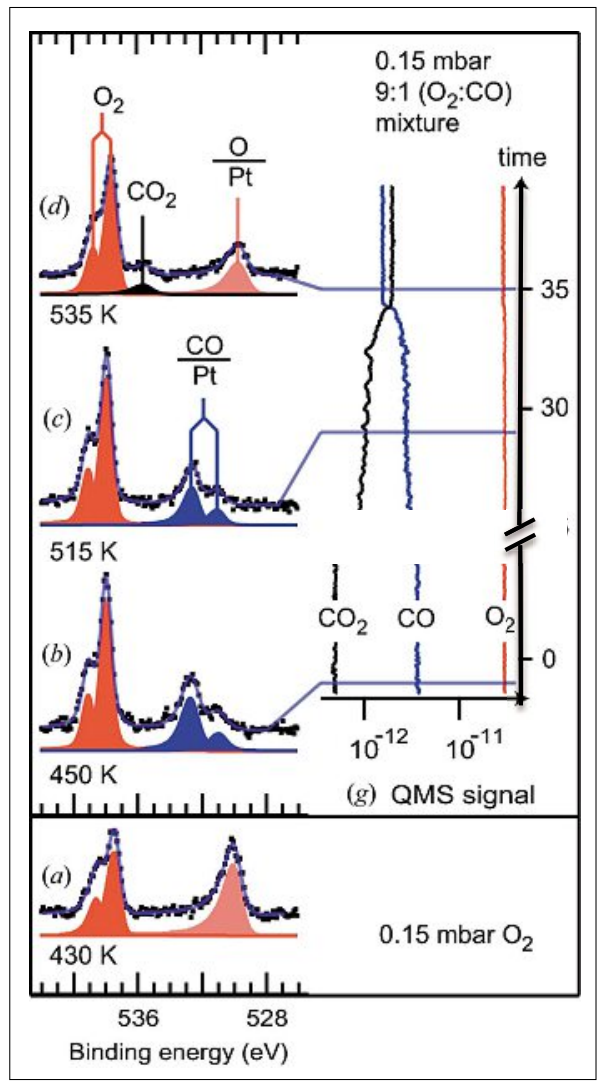

Fig. 15. (a) O1s photoelectron spectrum of a Pt (111) single crystal surface in $0.15 \mathrm{mbar}_{2}$ at $430 \mathrm{~K}$. (b)-(d) O1s photoelectron spectra in a 0.15 mbar 9:1 $\mathrm{O}_{2}: \mathrm{CO}$ mixture when the Pt crystal was heated from $450 \mathrm{~K}$ to $535 \mathrm{~K}$. The mass spectrometric gas analysis (QMS) of $\mathrm{CO}, \mathrm{O}_{2}$ and $\mathrm{CO}_{2}$ was performed simultaneously with the PES data for $35 \mathrm{~min}^{.12]}$ 
Pt surface. ${ }^{[12]}$ Initially the Pt surface was exposed to 0.15 mbar pure oxygen gas at $430 \mathrm{~K}$ ( $c f$. spectrum (a)). Under these conditions the $\mathrm{O} 1 \mathrm{~s}$ spectrum is dominated by a component at $530 \mathrm{eV}$ due to dissociated atomic oxygen adsorbed on the surface and two components at $537 \mathrm{eV}$ and $539 \mathrm{eV}$ assigned to molecular oxygen in the gas phase. Panels (b)-(d) show the O1s spectra as the temperature of the Pt crystal is raised while being simultaneously exposed to a $\mathrm{O}_{2}: \mathrm{CO}$ mixture of ratio $9: 1$. The signal from the adsorbed atomic oxygen immediately disappears and instead two signals appear due to molecular $\mathrm{CO}$ adsorbed at two different sites on the surface. As the temperature is raised above a critical point, $515 \mathrm{~K}$, the two $\mathrm{CO}$ signals disappear and are completely absent at $535 \mathrm{~K}$ marking the onset of $\mathrm{CO}_{2}$ production as also observed in the concomitant mass spectrometric analysis of the emitted gases ( $g$ ). At the same time, the signal due to adsorbed atomic oxygen reappears. Thus, a complete mapping of the catalytic process could be achieved, monitoring all relevant species.

\section{Final Remarks}

Photoelectron spectroscopy, rooted in the techniques of nuclear $\beta$-spectroscopy, has undergone a fascinating development over the past fifty years. This development has been driven to a large extent by concomitant parallel progress in other fields, such as in surface science, synchrotron radiation technology and molecular and condensed matter theoretical methods, involving intense cross-fertilization. The present article has focused on various aspects of materials for sustainable energy systems, however, the future scope of photoelectron spectroscopy is substantially wider and of great promise within large areas of application in physics, chemistry and biology.

\section{Acknowledgments}

This work has been sponsored by the Swedish Research Council (VR), the Swedish Energy Agency (STEM), the STandUpstrategic research program and the Knut and Alice Wallenberg Foundation.

\section{Received: December 5, 2014}

[1] H. Hertz, Annalen der Physik 1887, 31, 983.

[2] W. Hallwachs, Annalen der Physik 1888, 33, 301.

[3] P. Lenard, Annalen der Physik 1902, 8, 149.

[4] A. Einstein, Annalen der Physik 1905, 17, 132.

[5] J. A. Van den Akker, E. C. Watson, Phys. Rev. 1931, 37, 1631.

[6] K. Siegbahn, N. Svartholm, Nature 1946, 157, 872.

[7] K. Siegbahn, K. Edvarson, Nucl. Phys. 1956, 1, 137.

[8] K. Siegbahn, C. Nordling, A. Fahlman, R. Nordberg, K. Hamrin, J. Hedman, G. Johansson, T. Bergmark, S.-E. Karlsson, I. Lindgren, B. Lindberg, 'ESCA, atomic, molecular and solid state structure studied by means of electron spectroscopy', Nova Acta Regiae Soc. Sci. Upsaliensis, 1967, Ser. IV, Vol. 20.

[9] C. Nordling, S. Hagstrom, K. Siegbahn, Z Phys. 1964, 178, 433.

[10] K. Siegbahn, Nobel Lecture, 'Electron spectroscopy for atoms, molecules and condensed matter', Uppsala Univ., Sweden, Fysiska Institutionen, 1981.

[11] R. Ovsyannikov, P. Karlsson, M. Lundqvist, C. Lupulescu, W. Eberhardt, A. Fohlisch, S. Svensson, N. Martensson, J. Electron Spectrosc. Rel. Phenom. 2013, 191, 92.

[12] J. Schnadt, J. Knudsen, J. N. Andersen, H. Siegbahn, A. Pietzsch, F. Hennies, N. Johansson, N. Martensson, G. Ohrwall, S. Bahr, S. Mahl, O. Schaff, J. Synchrot. Radiat. 2012, 19, 701 .

[13] S. K. Eriksson, M. Hahlin, J. M. Kahk, I. J. Villar-Garcia, M. J. Webb, H. Grennberg, R. Yakimova, H. Rensmo, K. Edstrom, A. Hagfeldt, H. Siegbahn, M. O. M. Edwards, P. G. Karlsson, K. Backlund, J. Ahlund, D. J. Payne, Rev. Sci. Instrum. 2014, 85, 11.

[14] D. F. Ogletree, H. Bluhm, G. Lebedev, C. S Fadley, Z. Hussain, M. Salmeron, Rev. Sci. Instrum. 2002, 73, 3872.

[15] M. Salmeron, R. Schlogl, Surf. Sci. Rep. 2008, $63,169$.

[16] 'Recent Advances in Hard X-ray Photoeclectronspectroscopy (HAXPES)', Ed. W. Drube, J. Electron Spectrosc. Rel. Phenom. 2013, 190.

[17] B. Oregan, M. Grätzel, Nature 1991, 353, 737.

[18] A. Hagfeldt, G. Boschloo, L. C. Sun, L. Kloo, H. Pettersson, Chem. Rev. 2010, 110, 6595.
[19] U. Bach, D. Lupo, P. Comte, J. E. Moser, F. Weissortel, J. Salbeck, H. Spreitzer, M. Grätzel, Nature 1998, 395, 583.

[20] M. M. Lee, J. Teuscher, T. Miyasaka, T. N. Murakami, H. J. Snaith, Science 2012, 338, 643.

[21] M. Z. Liu, M. B. Johnston, H. J. Snaith, Nature 2013, 501, 395.

[22] L. Etgar, P. Gao, Z. S. Xue, Q. Peng, A. K. Chandiran, B. Liu, M. K. Nazeeruddin, M. Grätzel, J. Am. Chem. Soc. 2012, 134, 17396.

[23] S. A. Chambers, S. Thevuthasan, Y. J. Kim, G. S. Herman, Z. Wang, E. Tober, R. Ynzunza, J. Morais, C. H. F. Peden, K. Ferris, C. S. Fadley, Chem. Phys. Lett. 1997, 267, 51.

[24] L. Patthey, H. Rensmo, P. Persson, K. Westermark, L. Vayssieres, A. Stashans, A. Petersson, P. A. Bruhwiler, H. Siegbahn, S. Lunell, N. Martensson, J. Chem. Phys. 1999, 110,5913

[25] P. G. Karlsson, S. Bolik, J. H. Richter, B. Mahrov, E. M. J. Johansson, J. Blomquist, P. Uvdal, H. Rensmo, H. Siegbahn, A. Sandell, J. Chem. Phys. 2004, 120, 11224.

[26] R. Knut, R. Lindblad, S. Grachev, J. Y. Faou, M. Gorgoi, H. Rensmo, E. Sondergard, O. Karis, J. Appl. Phys. 2014, 115, 043714.

[27] J. Schnadt, A. Henningsson, M. P. Andersson, P. G. Karlsson, P. Uvdal, H. Siegbahn, P. A. Bruhwiler, A. Sandell, J. Phys. Chem. B 2004, 108,3114

[28] J. Schnadt, P. A. Bruhwiler, L. Patthey, J. N. O'Shea, S. Sodergren, M. Odelius, R. Ahuja, O. Karis, M. Bassler, P. Persson, H. Siegbahn, S. Lunell, N. Martensson, Nature 2002, 418, 620.

[29] J. M. Tarascon, M. Armand, Nature 2001, 414, 359.

[30] B. Philippe, R. Dedryvere, J. Allouche, F. Lindgren, M. Gorgoi, H. Rensmo, D. Gonbeau, K. Edstrom, Chem. Mater. 2012, 24, 1107.

[31] A. S. Arico, P. Bruce, B. Scrosati, J. M. Tarascon, W. Van Schalkwijk, Nat. Mater. 2005, 4, 366.

[32] R. A. Sharma, R. N. Seefurth, J. Electrochem. Soc. 1976, 123, 1763.

[33] S. Södergren, H. Rensmo, H. Siegbahn in ECASIA 97: 7th European Conference on Applications of Surface and Interface Analysis: Congress Centre, Göteborg, Sweden, June 16-20, 1997, Eds. I. Olefjord, L. Nyborg, D. Briggs, John Wiley \& Son Ltd., 1997.

[34] S. Malmgren, K. Ciosek, R. Lindblad, S. Plogmaker, J. Kuhn, H. Rensmo, K. Edstrom, M. Hahlin, Electrochim. Acta 2013, 105, 83.

[35] A. Henningsson, H. Rensmo, A. Sandell, H. Siegbahn, S. Sodergren, H. Lindstrom, A. Hagfeldt, J. Chem. Phys. 2003, 118, 5607.

[36] S. Malmgren, K. Ciosek, M. Hahlin, T. Gustafsson, M. Gorgoi, H. Rensmo, K. Edstrom, Electrochim. Acta 2013, 97, 23.

[37] H. Siegbahn, K. Siegbahn, J. Electron Spectrosc. Rel. Phenom. 1973, 2, 319. 